\section{New Mutants of Apolipoprotein E Associated with Atherosclerotic Diseases But Not to Type III Hyperlipoproteinemia}

Taku Yamamura, Akira Yamamoto, Tetsuya Sumiyoshi, Katsuhiko Hiramori, Yasuko Nishioeda, and Seiki Nambu Department of Etiology and Pathophysiology, and Department of Medicine, National Cardiovascular Center Research Institute, Suita, Osaka, 565 Japan

and four units of charge, respectively. While the apo ESuita isoprotein had the same molecular weight as ordinary major apo $\mathrm{E}$ isoproteins, the molecular weight of the apo E-5 isoprotein was 1,500-2,000 lower than the other apo $E$ isoproteins by sodium dodecyl sulfatepolyacrylamide gel electrophoresis.

The incidence of abnormal apo E components, including apo E-5 and apo E-Suita, was high among patients with hyperlipidemia and ischemic heart disease (7:127), while we could not find such components among 100 healthy individuals. Moreover, five of seven patients with the abnormal apo $\mathrm{E}$ had overt atherosclerotic disease. The findings suggest that these kinds of apolipoprotein mutation are closely related to the development of atherosclerosis.

\section{Introduction}

In recent years, it has been demonstrated that some structural alterations in apolipoproteins or their complete absence result in disturbances of plasma lipid and lipoprotein metabolism. Many such cases are of medical importance because they are associated with severe complications such as pancreatitis or atherosclerosis.

Type III hyperlipoproteinemia appears in individuals who are homozygous for the apolipoprotein $\mathrm{E}$ (apo E) ${ }^{1}$ mutation, apo E-2 $(1,2)$, or in those with a complete absence of apo E (3), when they have another hereditary factor or some nutritional factors which lead to combined hyperlipidemia $(1,2$, 4). Recently, another mutant of apo $E$ (apo $E_{\text {Bethesda }}$ ) associated with type III hyperlipoproteinemia was also reported (5). The association of an apo E mutation with hyperlipidemia was first demonstrated by Utermann et al. (6), and it was further shown by Mahley's group that the mutant, apo E-2, occurred by the substitution of cysteine for arginine as the 158th amino acid residue in the primary peptide structure of the wild-type form of apo E (apo E-3) $(7,8)$.

1. Abbreviations used in this paper: apo, apolipoprotein; apo $C$ and apo $\mathrm{E}$, apolipoproteins $\mathrm{C}$ and $\mathrm{E}$; $\mathrm{CCU}$, Coronary Care Unit; DDT dithiothreitol. 
Apo E-3 has an affinity for the apo B,E receptor on the cell surface, which has an important role in the regulation of cholesterol metabolism (9). Apo E-2, however, lacks such an affinity $(10,11)$. Other mutants also caused by point mutations in the amino acid sequence at places other than the residue 158 have been discovered by the same group $(12,13)$.

Another mutant of apo E, apo E-4, which is caused by a change at the residue 112 from cysteine to arginine $(7,8)$, has a very high frequency $(\sim 20 \%)$ among the general population (14-16). Although data have been presented showing an increased prevalence of apo E-4 in type $\mathrm{V}$ hyperlipoproteinemia (17), theoretical evidence is still lacking about the influence of this kind of mutation on lipoprotein metabolism.

Recently, we found a new component of apo E, designated apo E-5, which moved beyond E-IV ${ }^{2}$ to the cathodic side of an isoelectric focusing gel, and so, was more basic than apo E-4, in several patients with an atherosclerotic disease (18). In this paper, we describe another mutant, tentatively called apo ESuita, giving at least three bands upon isoelectric focusing, all of which were more basic than the ordinary apo E components, E-IV to E-I. Most of the probands with this mutation had an atherosclerotic disease. The prevalence of these newly found apo E mutants, including both apo E-5 and apo E-Suita, was as much as $5 \%$ among patients with atherosclerotic disease and hyperlipidemia, while we could not find any such abnormal apo $\mathrm{E}$ components among 100 healthy subjects. Therefore, a causal relation between these mutants and the development of atherosclerosis was strongly suggested. In addition, we have partially characterized these apo $\mathrm{E}$ mutants with respect to the nature of the alterations in their molecular structures.

\section{Methods}

Subjects. 69 patients ( 55 males and 14 females) who had been treated in the Coronary Care Unit (CCU) of our hospital, having been diagnosed as having angina pectoris or acute myocardial infarction, and 58 patients ( 37 males and 21 females) attending our lipid clinic with hyperlipidemia with or without atherosclerotic disease were the subjects of this study. We refer to the former subjects as CCU patients and to the latter as lipid clinic patients. 100 apparently healthy subjects (53 males and 47 females) were also studied as a control group. All individuals are unrelated. In some patients, family studies were performed and their available blood relatives and spouse were investigated.

Serum lipid and lipoprotein analysis. Blood samples were obtained after overnight fasting, and serum was separated after low-speed centrifugation at room temperature. Very low density lipoprotein (VLDL) was isolated from serum by a quantitative ultracentrifugation at a solvent density of $1.006 \mathrm{~g} / \mathrm{ml}$ as described previously (19). Cholesterol and triglycerides in serum and VLDL fractions were measured by enzymatic methods. High density lipoprotein (HDL)cholesterol concentration was determined in the supernatant after the

2. Bands of apo $E$ separated on an isoelectric focusing gel are designated as E-IV, E-III, E-II, E-I, and E-I' (from the cathodic to the anodic side) as reported by Utermann et al. $(1,6)$, and apo $E$ isoproteins, phenotypes, and genes are designated according to the classification proposed by Zannis et al. (32). precipitation of VLDL and low density lipoprotein (LDL) with heparin and calcium (20). The LDL-cholesterol was calculated by taking the difference between serum cholesterol and VLDL- plus HDL-cholesterol determined as above. The protein content of VLDL was determined by the method of Lowry et al. (21) using crystalline bovine serum albumin as standard. The turbidity of the reaction mixture was removed with Triton X-100 (22).

Isoelectric focusing and electrophoresis. VLDL apoproteins were obtained by acetone-ethanol $(1: 1, \mathrm{vol} / \mathrm{vol})$ delipidation of the ultracentrifugal VLDL fraction at $-20^{\circ} \mathrm{C}$. Apo $\mathrm{E}$ isoforms in apo VLDL were analyzed by isoelectric focusing on polyacrylamide gels. Analytical isoelectric focusing was performed according to the method of Warnick et al. (23) with minor modifications. Apo VLDL (300-350 $\mu \mathrm{g}$ protein) was solubilized in $200 \mu \mathrm{l}$ of $0.01 \mathrm{M}$ Tris- $\mathrm{HCl}$ (pH 8.6) containing 8.0 $\mathrm{M}$ urea and $0.01 \mathrm{M}$ dithiothreitol (DTT). The apolipoproteins were subjected to isoelectric focusing with $7.5 \%$ polyacrylamide gel rods $(0.5$ $\times 8.0 \mathrm{~cm}$ ) containing $8.0 \mathrm{M}$ urea and $2.0 \%$ carrier ampholytes. The Ampholine carrier ampholytes (LKB-Produkter AB, Bromma, Sweden), at $\mathrm{pH} 3.5-5$, $\mathrm{pH} 5-8$, and $\mathrm{pH} 3.5-10$, were mixed in proportions of 2:2:1 to create a $\mathrm{pH}$ gradient between 4 to 8 . To minimize the concentration of ammonium persulfate, gels were polymerized under ultraviolet light with the combination catalytic systems of riboflavin and ammonium persulfate. Focusing was carried out for $3,600 \mathrm{~V} \cdot \mathrm{h}$ at $10^{\circ} \mathrm{C}$. The proteins were fixed with $5 \%$ trichloroacetic acid and $5 \%$ sulfosalicylic acid, and stained with $0.1 \%$ Coomassie Brilliant Blue $\mathbf{R}$ 250. The gels were scanned at $570 \mathrm{~nm}$ with a densitometer. For determination of isoelectric points of apo $\mathrm{E}$ isoproteins, a gel without apolipoproteins was focused in parallel with the gels containing apolipoprotein samples and, then, cut into $5-\mathrm{mm}$ segments. $1 \mathrm{ml}$ of water was added to each gel segment and its $\mathrm{pH}$ was measured at room temperature. One-dimensional sodium dodecyl sulfate (SDS)-polyacrylamide slab gel electrophoresis was carried out on $12.5 \%$ polyacrylamide separating gels $(15 \times 14 \times 0.2 \mathrm{~cm})$ with $4 \%$ stacking gels $(2 \mathrm{~cm}$ in height) by the procedure of Laemmli et al. (24). The molecular weight of apo E was determined by one-dimensional SDS-polyacrylamide gel electrophoresis with the following molecular weight standards: bovine serum albumin, 68,000 ; ovalbumin, 43,000 ; chymotrypsinogen A, 26,000; soybean trypsin inhibitor, 21,000; myoglobin, 17,000; and cytochrome $c, 12,000$. Two-dimensional isoelectric focusing-SDS-polyacrylamide gel electrophoresis was performed by a modification of O'Farrell's method (25). For the first dimension, VLDL apoproteins dissolved in $0.01 \mathrm{M}$ Tris- $\mathrm{HCl}(\mathrm{pH} 8.6)$ containing $8.0 \mathrm{M}$ urea and $0.01 \mathrm{M}$ DTT were focused as described above. We used $5 \%$ polyacrylamide gel rods $(0.25 \times 8.0 \mathrm{~cm})$ as the first dimension and $12.5 \%$ polyacrylamide gel slabs $(15 \times 16 \times 0.2 \mathrm{~cm})$ as the second dimension. After electrophoresis, gels were stained with Coomassie Brilliant Blue R-250.

Characterization of apolipoproteins. Heparin-Sepharose CL-6B (Pharmacia Fine Chemicals AB, Uppsala, Sweden) affinity chromatography was performed according to the method of Shelburne and Quarfordt (26) with the previously described modification (18). Apolipoproteins bound to the gel in the low salt $(0.05 \mathrm{M} \mathrm{NaCl})$ buffer were eluted with the high salt $(0.5 \mathrm{M} \mathrm{NaCl})$ buffer. The bound and unbound fractions were analyzed by both isoelectric focusing and SDSpolyacrylamide gel electrophoresis. Immunochemical characterization of apolipoproteins was carried out as follows. Analytical isoelectric focusing of apo VLDL was performed in duplicate on $5 \%$ polyacrylamide gel rods $(0.25 \times 8.0 \mathrm{~cm})$. One of a pair of gels was stained with Coomassie Brilliant Blue R-250. The other gel was incubated twice in $15 \mathrm{ml}$ of $0.01 \mathrm{M}$ Tris- $\mathrm{HCl}$ (pH 8.2) containing 2.0\% Triton X-100 for 
Table I. Clinical and Biochemical Characteristics of the Probands with Apo E-5 or Apo E-Suita

\begin{tabular}{|c|c|c|c|c|c|c|}
\hline \multirow[b]{2}{*}{ Case } & \multirow[b]{2}{*}{ Age } & \multirow[b]{2}{*}{ Sex } & \multicolumn{2}{|c|}{ Serum lipid* } & \multirow{2}{*}{$\begin{array}{l}\text { Apo E } \\
\text { phenotype }\end{array}$} & \multirow[b]{2}{*}{ Clinical findings } \\
\hline & & & Ch & TG & & \\
\hline & $y r$ & & & & & \\
\hline 1. K.N. & 37 & $\mathbf{M}$ & 316 & 48 & E4/Suita & Cerebral infarction, angina pectoris, hypertension \\
\hline 2. Y.F. & 57 & $\mathbf{M}$ & 311 & 121 & E3/Suita & Myocardial infarction, diabetes mellitus \\
\hline 3. H.M. & 74 & $\mathbf{F}$ & 188 & 146 & E3/Suita & Myocardial infarction, cerebral infarction \\
\hline 4. $\mathrm{S} . \mathrm{Og}$. & 40 & $\mathbf{M}$ & 271 & 586 & E4/Suita & Chronic hepatitis, diabetes mellitus (mild) \\
\hline 5. S.K. $\ddagger$ & 50 & $\mathbf{M}$ & 278 & 135 & E5/3 & Myocardial infarction \\
\hline 6. K.O.‡ & 65 & $\mathbf{M}$ & 265 & 226 & E5/3 & Multiple cerebral infarction \\
\hline 7. T.Y.‡ & 49 & $\mathbf{M}$ & 219 & 132 & $\mathrm{E} 5 / 4$ & Chest pain, liver disease \\
\hline
\end{tabular}

* Ch, cholesterol (milligrams per deciliter); TG, triglycerides (milligrams per deciliter). $¥$ Concentration of lipoprotein lipids and family studies have been reported in reference (18).

15 min and embedded in a $1.0 \%$ agarose gel plate containing monospecific antibody against ordinary human apo $\mathrm{E}$ (E-4 to E-2). The agarose gel plate was left standing for $48 \mathrm{~h}$ at room temperature, and washed in $0.9 \% \mathrm{NaCl}$ for $3 \mathrm{~d}$. Immunoprecipitate was then stained with Coomassie Brilliant Blue R-250.

Chemical and enzymatic modification of VLDL. To determine the number of cysteine residues and to estimate charge unit differences between each apolipoprotein component, VLDL was treated with cysteamine (Sigma Chemical Co., St. Louis, MO) as described by Weisgraber et al. (7). Ultracentrifugal VLDL fractions were dialyzed overnight against water and lyophilized. Cysteamine was added to VLDL samples dissolved in $0.1 \mathrm{M} \mathrm{NH}_{4} \mathrm{HCO}_{3}$. The mixture was incubated for $4 \mathrm{~h}$ at $37^{\circ} \mathrm{C}$ and then lyophilized. The treated samples were delipidated and dissolved in the focusing sample buffer without DTT, and subjected to analytical isoelectric focusing. Control samples were incubated in parallel with treated samples, and analyzed by our routine focusing procedure. For determination of sialylated apo E isoforms, VLDL samples were treated with neuraminidase (from Clostridium perfringens, type VI, Sigma Chemical Co.). Lyophilized VLDL was dissolved in $0.1 \mathrm{M}$ acetate buffer (pH 5.0), and incubated with $0.1 \mathrm{U}$ neuraminidase/mg VLDL protein for $2.5 \mathrm{~h}$ at $37^{\circ} \mathrm{C}$. Controls were incubated in acetate buffer alone. After treatment, the samples were delipidated and subjected to isoelectric focusing or twodimensional electrophoresis.

\section{Results}

Based on the isoelectric focusing patterns of apo VLDL, six major apo $\mathrm{E}$ phenotypes were detected which were made up by a combination of the three major apo $\mathrm{E}$ isoproteins. Besides these major phenotypes, a minor one including apo E-5 has been detected in three patients (18), and in addition, we discovered another phenotype with a new series of apolipoprotein components, apo E-Suita, in four patients with hyperlipidemia and atherosclerotic disease (Table I).

Typical isoelectric focusing patterns of apo VLDL with apo E-Suita are shown in Fig. 1. Besides one of the already known major apo E components, E-IV to E-II, accompanied by some minor bands of its sialylated derivatives, each subjects had at least three bands in the region corresponding to E-VII, E-VI, and E-V. The major apo E bands (E-IV, E-III, and EII) had apparent isoelectric points of 5.91, 5.78, and 5.68 in our focusing system, and the isoelectric points of the E-VII, E-VI, and E-V bands were $6.89,6.35$, and 6.08 , respectively.

The frequency distribution of the different apo $\mathrm{E}$ phenotypes in the three groups of this study is shown in Table II. The patterns of distribution of six common apo $\mathrm{E}$ phenotypes, E4/4 to E2/2, in the groups of the lipid clinic patients and the CCU patients were similar to that in the group of healthy individuals. An E2/2 phenotype was only found in the lipid

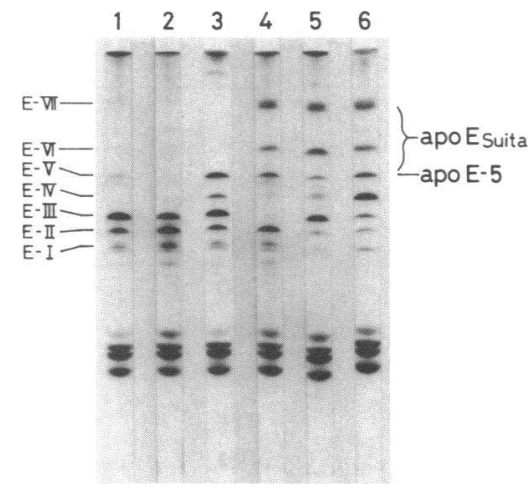

Figure 1. Various apo E phenotypes separated by isoelectric focusing (pH gradient 3.5-8.0) of apo VLDL. The E-V band shown on gel 3 and the E-VII to E-V bands on gel 4 to 6 are the new apolipoprotein components, apo E-5 and apo E-Suita, respectively, investigated in this study. The apo E phenotypes are shown: (1) E3/3, (2) E3/2, (3) E5/3, (4) E2/Suita, (5) E3/Suita, and (6) E4/Suita. Four apoprotein bands in the lower portion of each gel correspond to apo C-III-0, apo C-II, apo C-III-1, and apo C-III-2 from top to bottom. The cathode (basic) is at the top and the anode (acidic) at the bottom. 
Table II. Apo E Phenotype Frequency in the Three Groups of Subjects in this Study

\begin{tabular}{|c|c|c|c|c|c|c|}
\hline \multirow{2}{*}{$\begin{array}{l}\text { Apo E } \\
\text { phenotype }\end{array}$} & \multicolumn{2}{|c|}{$\begin{array}{l}\text { Lipid clinic } \\
\text { patients* }\end{array}$} & \multicolumn{2}{|c|}{ CCU patientsł } & \multicolumn{2}{|c|}{ Healthy subjects§ } \\
\hline & No. & Percent & No. & Percent & No. & Percent \\
\hline$E 4 / 4$ & 0 & (0) & 1 & (1.4) & 1 & (1.0) \\
\hline $\mathrm{E} 4 / 3$ & 8 & (13.8) & 15 & (21.8) & 15 & $(15.0)$ \\
\hline $\mathrm{E} 4 / 2$ & 1 & $(1.7)$ & 1 & (1.4) & 1 & $(1.0)$ \\
\hline $\mathrm{E} 3 / 3$ & 34 & (58.6) & 45 & (65.3) & 71 & (71.0) \\
\hline $\mathrm{E} 3 / 2$ & 7 & (12.1) & 4 & $(5.8)$ & 12 & (12.0) \\
\hline $\mathrm{E} 2 / 2$ & 4 & $(6.9)$ & 0 & (0) & 0 & $(0)$ \\
\hline E4/Suita & 2 & & 0 & & 0 & \\
\hline E3/Suita & 0 & & 2 & & 0 & \\
\hline E5/4 & 1 & & 0 & & 0 & \\
\hline $\mathrm{E} 5 / 3$ & 1 & & 1 & & 0 & \\
\hline Subtotal & 4 & (6.9) & 3 & (4.3) & 0 & (0) \\
\hline Total & 58 & $(100)$ & 69 & $(100)$ & 100 & $(100)$ \\
\hline
\end{tabular}

* 58 patients ( 37 males and 21 females) attending our lipid clinic with hyperlipidemia and with or without atherosclerotic disease.

$¥ 69$ patients ( 55 males and 14 females) treated in the CCU having angina pectoris or acute myocardial infarction.

$\S 100$ apparently healthy individuals ( 53 males and 47 females).

clinic patients. Serum lipoprotein patterns of the subjects with the E2/2 phenotype showed the characteristics of classical type III hyperlipoproteinemia or broad- $\beta$ disease. The newly found apo $\mathrm{E}$ mutants including both apo E-5 and apo E-Suita, however, were found in four subjects of 58 lipid clinic patients (6.9\%) and in three of $69 \mathrm{CCU}$ patients (4.3\%). On the contrary, we could not find any such abnormal apo E components among 100 healthy subjects (Table II). In addition, five of these seven subjects with abnormal apo E, apo E-5, or apo E-Suita, had overt atherosclerotic diseases (Table I). Family members in some patients with abnormal apo $\mathrm{E}$ were also investigated. Data on the study of the patients with apo E-5 have been reported in our previous paper (18). Table III and Fig. 2 show serum lipoproteins and apo $E$ phenotypes in the patients with apo E-Suita and their relatives. In the N-kindred, both the proband and his brother had an E4/Suita phenotype. The proband of the F-kindred had the apo E phenotype of E3/Suita, and his spouse E3/2. One of the daughters was demonstrated to have an E2/Suita phenotype, and the other E3/3 (Fig. 2, Table III). None of the subjects with apo E-5 or apo E-Suita had cholesterol-rich VLDL or the broad- $\beta$ pattern of serum lipoproteins, the characteristics of type III hyperlipoproteinemia (data not shown).

Immunochemical examination of the isoelectric focusing gels with anti-apo $\mathrm{E}$ antibody revealed that apo E-Suita, as well as apo E-4, apo E-3, and their sialylated derivatives, gave an immunochemical precipitation reaction with antibody prepared with the ordinary apo E components (Fig. 3). To determine whether these apo E-Suita components bind to heparin like the already known apo $\mathrm{E}$ isoproteins (26), fractionation of apo VLDL by heparin-Sepharose affinity chromatography was performed. Apo E-Suita was not eluted from a column of heparin-Sepharose CL-6B with a low salt buffer,

Table III. Concentration of Serum and Lipoprotein Lipids, and Apo E Phenotypes in the Family Members in Apo E-Suita Kindreds

\begin{tabular}{|c|c|c|c|c|c|c|c|c|c|}
\hline \multirow[b]{2}{*}{ Subject } & \multirow[b]{2}{*}{ Age } & \multirow[b]{2}{*}{ Sex } & \multicolumn{2}{|c|}{ Serum } & \multicolumn{2}{|c|}{ VLDL } & \multirow{2}{*}{$\frac{\mathrm{LDL}}{\mathrm{Ch}^{*}}$} & \multirow{2}{*}{$\frac{\mathrm{HDL}}{\mathrm{Ch}^{*}}$} & \multirow{2}{*}{$\begin{array}{l}\text { Apo E } \\
\text { phenotype }\end{array}$} \\
\hline & & & $\mathrm{Ch}^{*}$ & TG* $^{*}$ & $\mathrm{Ch}^{*}$ & $\mathrm{TG}^{*}$ & & & \\
\hline & $y r$ & & & & & & & & \\
\hline \multicolumn{10}{|l|}{ N-kindred } \\
\hline Proband & 37 & $\mathbf{M}$ & 316 & 48 & 3 & 8 & 298 & 15 & E4/Suita \\
\hline Spouse & 39 & $\mathbf{F}$ & 198 & 107 & 16 & 53 & 140 & 42 & E3/3 \\
\hline Brother & 32 & $\mathbf{M}$ & 350 & 87 & 16 & 33 & 296 & 38 & E4/Suita \\
\hline Son & 10 & $\mathbf{M}$ & 246 & 136 & 22 & 90 & 164 & 60 & $\mathrm{E} 4 / 3$ \\
\hline \multicolumn{10}{|l|}{ F-kindred } \\
\hline Proband & 57 & $\mathbf{M}$ & 311 & 121 & 11 & 46 & 262 & 38 & E3/Suita \\
\hline Spouse & 54 & $\mathbf{F}$ & 194 & 180 & 37 & 126 & 123 & 34 & $\mathrm{E} 3 / 2$ \\
\hline Daughter & 23 & $\mathbf{F}$ & 206 & 82 & 10 & 43 & 158 & 38 & E2/Suita \\
\hline Daughter & 21 & $\mathbf{F}$ & 139 & 62 & 6 & 33 & 94 & 39 & $E 3 / 3$ \\
\hline \multicolumn{10}{|l|}{ M-kindred } \\
\hline Proband & 74 & $\mathbf{F}$ & 188 & 146 & 21 & 75 & 136 & 31 & E3/Suita \\
\hline \multicolumn{10}{|l|}{ Og-kindred } \\
\hline Proband & 40 & $\mathbf{M}$ & 271 & 586 & 165 & 455 & 80 & 26 & E4/Suita \\
\hline
\end{tabular}

* Ch, cholesterol (milligrams per deciliter); TG, triglycerides (milligrams per deciliter). 


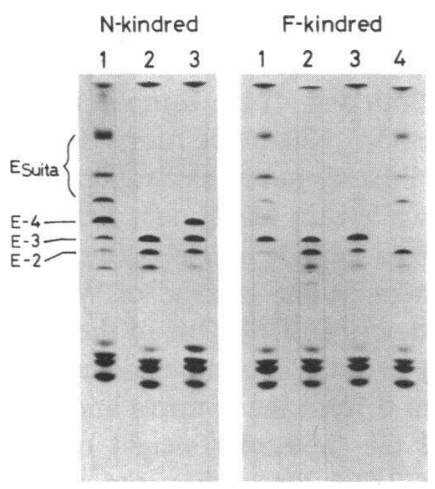

Figure 2. Isoelectric focusing patterns of apo VLDL from the probands and family members of two kindreds with apo E-Suita. N-kindred: (1) apo VLDL from the proband with phenotype E4/Suita, (2) apo VLDL from his spouse with phenotype E3/3, and (3) apo VLDL from the son with phenotype E4/3. F-kindred: (1) apo VLDL from the proband with E3/Suita, (2) apo VLDL from his spouse with E3/2, (3) apo VLDL from a daughter with E3/3, and (4) apo VLDL from another daughter with E2/Suita. The cathode is at the top and the anode at the bottom.

but was eluted with a high salt buffer together with other apo E components as a bound fraction. Apoproteins in the bound fraction showed a single band coupled with ordinary apo $\mathrm{E}$ by one-dimensional SDS-polyacrylamide gel electrophoresis (data not shown). As previously described (18), apo E-5 had the same characteristics as apo E-Suita with respect to the interaction with heparin and to the reaction with the anti-apo $\mathrm{E}$ antibody.

It has been shown that the presence of cysteine residues in a protein can be detected by monitoring the charge in the isoelectric focusing pattern after modification with cysteamine, and that cysteamine treatment gives the protein a single positive charge per one cysteine residue (7). After cysteamine treatment of VLDL, both apo E-5 (E-V and E-IV bands) and apo E-Suita (E-VII to E-IV bands) were shifted to higher isoelectric points by a single charge unit as apo E-3, which contained one residue of cysteine per mole (Fig. 4). These

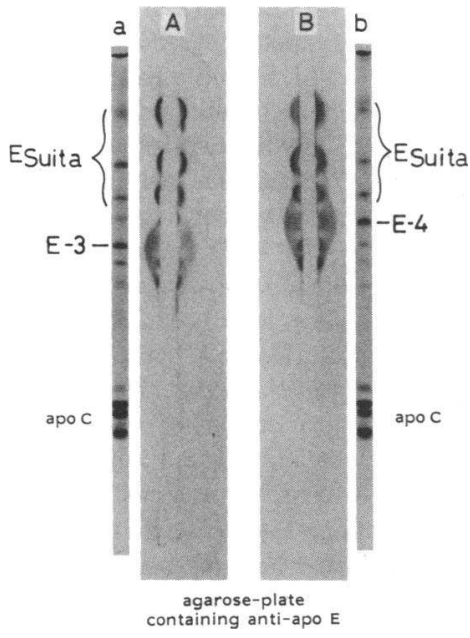

Figure 3. Examination of apo VLDL from two patients with apo E-Suita $[(A)$ the proband of F-kindred and $(B)$ the proband of $\mathrm{N}$ kindred shown in Fig. 2] with combined gel isoelectric focusing and an immunodiffusion system. After duplicate focusing of apo VLDL, one of the pair of gels was embedded in a $1 \%$ agarose gel plate which contained anti-apo E antibodies. Immunoprecipitate was stained with Coomassie Brilliant Blue R-250. Gels $(a)$ and $(b)$, which were stained with Coomassie

Brilliant Blue directly after focusing, show the remaining one of the pair of gels, corresponding to gels $(A)$ and $(B)$, respectively.

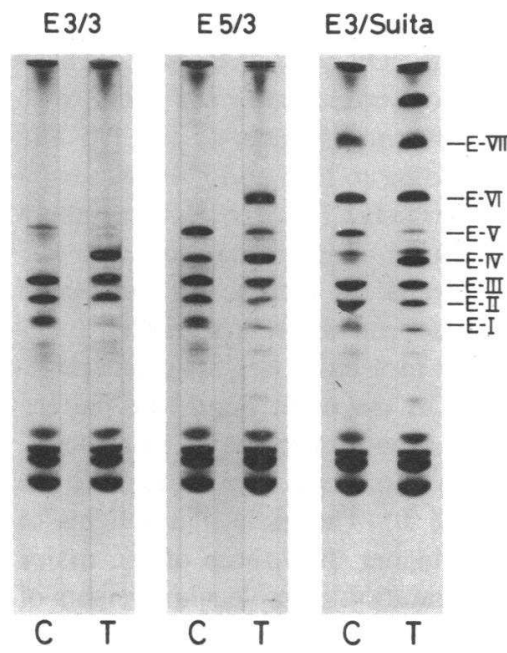

Figure 4. Effect of cysteamine treatment on the isoelectric focusing pattern of apo VLDL. Left: control $(C)$ and cysteamine-treated $(T)$ apo VLDL from a subject with the E3/3 phenotype; center: $C$ and $T$ apo VLDL from a patient with the E5/3 phenotype; and right: $C$ and $T$ apo VLDL from a patient with the E3/Suita phenotype. Both apo E-5 (E-V and E-IV) and apo E-Suita (E-VII to E-IV) components are shifted to higher isoelectric points by a single charge unit as apo E-3 components (E-III to E-I). The cathode is at the top and the anode at the bottom. Four apoprotein bands in the lower portion of each gel correspond to apo $\mathrm{C}$-II and apo $\mathrm{C}$-III isoforms.

results indicate the presence of one cysteine residue in the apo E-5 and apo E-Suita polypeptide chains.

Apo VLDL from the patients with the E5/3 phenotype

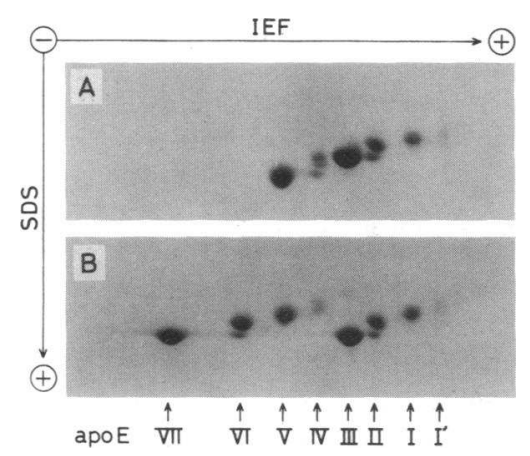

Figure 5. Two-dimensional electrophoresis of apo VLDL from a patient with the E5/3 phenotype $(A)$ and from a patient with the E3/ Suita phenotype $(B)$. Only the part of gel containing apo E components is shown. Note that distribution patterns similar to apo E-3 and its sialylated isoforms (E-III to E-I) are observed for apo E-5 (E$\mathrm{V}$ and E-IV) and apo E-Suita components (E-VII to E-IV). Apparent molecular weights of two major isoforms from the E3/Suita phenotype, corresponding to E-VII and E-III, are similar by SDS-electrophoresis. However, there was a difference in the apparent molecular weight between major isoforms from the $\mathrm{E} 5 / 3$ phenotype, corresponding to E-V and E-III. "IEF" means isoelectric focusing for the first dimension, and "SDS" means SDS-electrophoresis for the second dimension. 
and with the E3/Suita phenotype were analyzed by twodimensional isoelectric focusing-SDS-polyacrylamide gel electrophoresis (Fig. 5). The electrophoretic pattern of apo E with the E5/3 phenotype showed two major spots of apo E-5 and apo E-3 isoproteins, corresponding to E-V and E-III bands on one-dimensional isoelectric focusing. The apo E-5 isoprotein had a lower apparent molecular weight than the apo E-3 isoprotein on SDS-electrophoresis for the second dimension (Fig. $5 \mathrm{~A}$ ). The other minor isoproteins, corresponding to $\mathrm{E}$ IV and to E-II-E-I', had higher molecular weights than apo E5 and apo E-3, respectively. On the other hand, the pattern from the E3/Suita phenotype showed two major isoproteins, E-VII and E-III, and these two isoproteins seemed to have the same molecular weights (Fig. $5 \mathrm{~B}$ ). The molecular weights of other minor isoproteins were higher than those of the major isoproteins. For the precise estimation of molecular weights of the major apo E isoproteins, one-dimensional SDS-polyacrylamide slab gel electrophoresis with molecular weight markers was carried out using the discontinuous buffer system by the Laemmli's procedure (Fig. 6). Control apo VLDL samples with E4/2 and E3/3 phenotypes showed a single major apo $E$ band with an approximate molecular weight of 35,000 , determined by the molecular weight standards, and a minor band of apo $\mathrm{E}$ with a relatively higher molecular weight (Fig. 6, lanes 1 and 4). Similar patterns of apo $E$ from two patients

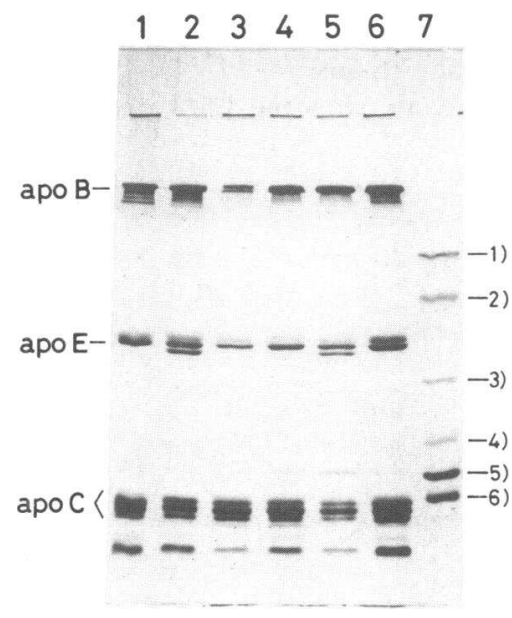

Figure 6. One-dimensional SDS-polyacrylamide gel electrophoresis of apo VLDL from control subjects with apo $E$ phenotypes of E4/2 and E3/3 (lanes 1 and 4, respectively), apo VLDL from probands with the phenotype E5/3 in K-kindred and in O-kindred (lanes 2 and 5 , respectively), apo VLDL from probands with the phenotype E3/Suita in F-kindred and in M-kindred (lanes 3 and 6, respectively), and the molecular weight standard with different marker proteins (lane 7). The numbers of the molecular weight standard are shown: 1) bovine serum albumin, 68,$000 ; 2$ ) ovalbumin, 43,$000 ; 3$ ) chymotrypsinogen A, 26,$000 ; 4)$ soybean trypsin inhibitor, 21,000 ; 5) myoglobin, 17,000 ; and 6) cytochrome $c, 12,000$. Note that apo $\mathrm{E}$ from the patients with the E5/3 phenotype was composed of two major bands. with the E3/Suita phenotype (cases 2 and 3) were observed with a major apo $\mathrm{E}$ band at the apparent molecular weight of 35,000 (Fig. 6, lanes 3 and 6). Both apo VLDL samples from two unrelated patients with the E5/3 phenotype (cases 5 and 6), however, had two major apo $\mathrm{E}$ bands in this electrophoretic system (Fig. 6, lanes 2 and 5), while they had shown a broad single band of apo E on SDS-polyacrylamide gel electrophoresis using the continuous buffer system by the method of Weber and Osborn (27), as previously described (18). One of the two bands in cases 5 and 6 (Fig. 6) revealed the same molecular weight $(35,000)$ as the major apo $\mathrm{E}$ from the controls and the patients with apo E-Suita, and the molecular weight of an additional one was $\sim 1,500$ to 2,000 lower than the other major apo E components.

We investigated sialylated isoforms of apo E-Suita by the two-dimensional electrophoresis after neuraminidase treatment of VLDL from the subject with the E3/Suita phenotype (Fig. 7). The intensity of the two major apo $E$ isoproteins appeared to be unchanged after treatment with neuraminidase, whereas the minor apo $\mathrm{E}$ isoproteins, having relatively higher molecular weights and more acidic isoelectric points, had disappeared or markedly diminished. Similar effects of neuraminidase treatment on isoelectric focusing patterns of apo $\mathrm{E}$ were observed in the VLDL from the patient with apo E-5 (data not shown).

\section{Discussion}

We analyzed the heterogeneity of apo E in VLDL from 58 hyperlipidemic subjects with or without atherosclerosis, 69 patients with ischemic heart disease, and 100 apparently

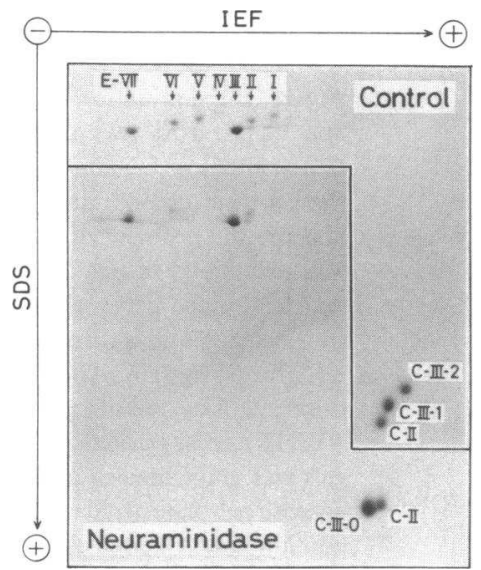

Figure 7. Effect of neuraminidase treatment upon two-dimensional electrophoretic patterns of apo VLDL from a patient with the E3/Suita phenotype. Only the parts of gel containing both apo $\mathrm{E}$ and apo C components are shown. After VLDL samples were incubated with (neuraminidase, lower panel) or without (control, upper panel) Clostridium perfringens neuraminidase, they were delipidated and subjected to two-dimensional electrophoresis. Note that minor components of apo E (E-VI to E-IV, E-II, and E-I) as well as apo C-III-2 and apo C-III-1 have disappeared or markedly diminished, and the intensity of apo C-III-O have increased following neuraminidase treatment. "IEF" means isoelectric focusing for the first dimension, and "SDS" means SDS-electrophoresis for the second dimension. 
healthy individuals. Six common apo E phenotypes, resulting from three major apo $\mathrm{E}$ alleles $(\epsilon 4, \epsilon 3$, and $\epsilon 2)$, were demonstrated on one-dimensional isoelectric focusing gels $(15,16$, 18). Gene frequencies of apo E alleles, which were calculated from the phenotype distribution in unrelated healthy individuals (Table II), were as follows: $\epsilon 4,0.090 ; \epsilon 3,0.845$; and $\epsilon 2,0.065$. Utermann et al. $(14,16)$ reported the gene frequencies in Germany as $\epsilon 4,0.150 ; \epsilon 3,0.773$; and $\epsilon 2,0.077$, and Zannis and Breslow (15) indicated those in the United States to be $0.11,0.72$, and 0.17 , respectively. From these results, it was suggested that there were no striking differences in apo $E$ allele frequencies among different populations. In addition, the distributions of six common apo $\mathrm{E}$ phenotypes in the groups of lipid clinic patients and CCU patients were similar to the distribution in the group of healthy subjects. Though the prevalence of the E2/2 phenotype was as much as $7 \%$ in the lipid clinic patients (Table II), this was due to the artificial clustering of type III hyperlipoproteinemia in the clinic. The expected frequency of the E2/2 phenotype from the observed $\epsilon 2$ frequency is $0.4 \%$ on the assumption of Hardy-Weinberg's equilibrium.

In this study, the new series of apo E components, apo E-Suita, was discovered in four patients. Two of them suffered from myocardial infarction, and both had mixed-lipoproteintype hyperlipidemia or combined hyperlipidemia (cases 2 and 3). Another patient (case 1) had familial hypercholesterolemia. $\mathrm{He}$ also had a history of hypertension and suffered from cerebral infarction at the age of 35 . The last patient (case 4) had hyperlipoproteinemia type IV or type V and responded well to dietary restriction. We found the abnormal components, apo E-5 and apo E-Suita, in 7 out of 127 subjects with hyperlipidemia and/or atherosclerotic disease. It is especially noteworthy that three of these seven were found among patients with definite signs of ischemic heart disease being treated in the CCU, and that other two patients had also overt atherosclerotic disease (Table I). We could not find either apo E-5 nor apo E-Suita among 100 apparently healthy subjects. Therefore, it seems very likely that these kinds of apolipoprotein mutation are closely related to the development of atherosclerosis. However, most of the patients had combined hyperlipidemia instead of type III hyperlipoproteinemia, so the mechanism of the development of atherosclerosis must be independent of the retention of $\beta$-VLDL or of the cholesterol-rich remnant $(9,28)$. The prevalence of these newly found apo $E$ mutants in atherosclerotic and hyperlipidemic subjects may be limited within the Japanese population because these kinds of mutants have never been reported from Western countries either in patients with ischemic heart disease or in population studies $(14-16,29)$.

If we assume that the combination of new components, apo E-Suita, is determined by inheritance of a new apo E allele ( $\epsilon$ Suita) which is located at the same genetic locus as the already known apo $\mathrm{E}$ components, the apo $\mathrm{E}$ phenotype distribution among family members could be well explained by the current genetic model concerning the heterogeneity of human apo $\mathrm{E}(15,16)$. With other interpretations, it is difficult to explain the apo $\mathrm{E}$ phenotypes especially in the F-kindred (Fig. 2). Apo E-5 was controlled by an additional apo E allele $(\epsilon 5)$ at the same locus (18). All the probands with apo E-Suita had hyperlipidemia. However, the causal relationship between the apo E-Suita and hyperlipidemia is unclear, for one of the daughters of case 2 (F-kindred), who has an apo E phenotype of E2/Suita, had neither hyperlipoproteinemia nor cholesterolrich VLDL (Table III).

The components of apo E-Suita were identical with ordinary apo E components with respect to their interaction with heparin-Sepharose gel (26) as well as their interaction with monospecific antibodies against human apo E. As shown by Zannis and Breslow (15), each apo $\mathrm{E}$ component with a definite amino acid sequence appears in two or three forms due to posttranslational modification with sialic acid. The modified proteins are shifted to a more acidic isoelectric point, i.e., they move to the anodic side upon isoelectric focusing. It was suggested that the most basic among the three or four bands of apo E-Suita corresponding to E-VII was the unsialylated form, and that the other less basic components are products of posttranslational modification. This has been confirmed by the results of two-dimensional electrophoresis and neuraminidase treatment. The components on the acidic side of the E-VII component had higher molecular weights than E-VII on the two-dimensional electrophoresis, and had disappeared or diminished after the treatment with neuraminidase. Apo E-5 corresponding to E-V has also been identified to be the unsialylated isoprotein as previously suggested (18).

Charge modification with cysteamine indicated that both apo E-5 and apo E-Suita contained a single cysteine residue in their polypeptide chains. Furthermore, the observations on cysteamine treatment and the findings of unsialylated and sialylated isoforms showed that the unsialylated apo E-5 and apo E-Suita were shifted by two and four positive charge units, respectively, compared with the unsialylated apo E-3. The parent type of both apo E-5 and apo E-Suita can not be apo E-4, but they are probably formed as a result of substitutions of amino acids in the apo E-3 polypeptide because one cysteine residue is present in apo E-5 and apo E-Suita as in apo $E-3$, and because $\epsilon 3$, which is responsible for the production of apo E-3, is the most common allele in the general population.

It had been shown that apo E-4 and apo E-2 differed from apo E-3 by a single amino acid substitution at residue 112 (arginine for cysteine) and at residue 158 (cysteine for arginine), respectively $(7,8)$. Recently, Rall et al. reported minor mutants of apo E, i.e., apo E-2* (12) and apo E-2** (13), which resulted from the substitution of a cysteine residue for arginine at residue 145, and from the substitution of a glutamine residue for lysine at residue 146 , respectively. All these mutants of apo $\mathrm{E}$ were due to a single amino acid substitution. Substitution of an amino acid residue with a negative charge for another one with a positive charge can result in a relative 
increase of positive charges for the substituted protein by two units. However, it is unlikely that this kind of amino acid substitution is responsible for the change from apo E-3 to E-5 because there was a significant decrease in an apparent molecular weight. Deletion of a peptide fragment rich in acidic amino acid residues seems to be responsible for the mutation. In contrast, apo E-Suita had an apparent molecular weight similar to the common apo E, whereas it was shifted as much as four positive charge units compared with apo E-3. Peptide sequence analysis is now going on in our laboratory.

Mahley and his co-workers showed that the special domain containing the residues $139-146$ of an apo E polypeptide chain was important for its binding to apo B,E receptors $(30,31)$, and that the mutants of apo E-2, having amino acid mutations in this region, were defective in receptor binding (10-13). Receptor binding activity must be preserved in our new mutant apo $\mathrm{E}$ because the mutations were not associated with the retention of $\beta$-VLDL or cholesterol-rich remnant. Detailed structural and functional analyses of our mutants must be performed in the future, especially with respect to the association with hyperlipidemia and atherosclerosis.

\section{Acknowledgments}

We wish to thank Ms. Keiko Fujikawa and Ms. Yuko Saga for their excellent technical assistance.

This work was supported in part by a Grant-in-Aid for Scientific Research No. 58480151 from the Japanese Ministry of Education, Science and Culture, and by a Grant-in-Aid (1983) from Research Foundation for Cancer and Cardiovascular disease, Japan.

\section{References}

1. Utermann, G., M. Hees, and A. Steinmetz. 1977. Polymorphism of apolipoprotein E and occurrence of dysbetalipoproteinaemia in man. Nature (Lond.). 269:604-607.

2. Utermann, G., K. H. Vogelberg, A. Steinmetz, W. Schoenborn, N. Pruin, M. Jaeschke, M. Hees, and H. Canzler. 1979. Polymorphism of apolipoprotein E. II. Genetics of hyperlipoproteinemia type III. Clin. Genet. 15:37-62.

3. Ghiselli, G., E. J. Schaefer, P. Gascon, and H. B. Brewer, Jr. 1981. Type III hyperlipoproteinemia associated with apolipoprotein $\mathrm{E}$ deficiency. Science (Wash. DC). 214:1239-1241.

4. Hazzard, W. R., G. R. Warnick, G. Utermann, and J. J. Albers. 1981. Genetic transmission of isoapolipoprotein $\mathrm{E}$ phenotypes in a large kindred: relationship to dysbetalipoproteinemia and hyperlipidemia. Metab. Clin. Exp. 30:79-88.

5. Gregg, R. E., G. Ghiselli, and H. B. Brewer, Jr. 1983. Apolipoprotein $E_{\text {Bethecthe }}$ a new variant of apolipoprotein $E$ associated with type III hyperlipoproteinemia. J. Clin. Endocrinol. Metab. 57:969974.

6. Utermann, G., M. Jaeschke, and J. Menzel. 1975. Familial hyperlipoproteinemia type III: deficiency of a specific apolipoprotein (apo E-III) in the very-low-density lipoproteins. FEBS (Fed. Eur. Biochem. Soc.) Lett. 56:352-355.
7. Weisgraber, K. H., S. C. Rall, Jr., and R. W. Mahley. 1981. Human $\mathrm{E}$ apoprotein heterogeneity: cysteine-arginine interchanges in the amino acid sequence of the apo-E isoforms. J. Biol. Chem. 256:9077-9083.

8. Rall, S. C., Jr., K. H. Weisgraber, and R. W. Mahley. 1982. Human apolipoprotein E: the complete amino acid sequence. J. Biol. Chem. 257:4171-4178.

9. Mahley, R. W. 1982. Atherogenic hyperlipoproteinemia: the cellular and molecular biology of plasma lipoproteins altered by dietary fat and cholesterol. Med. Clin. N. Am. 66:375-402.

10. Weisgraber, K. H., T. L. Innerarity, and R. W. Mahley. 1982. Abnormal lipoprotein receptor-binding activity of the human $\mathrm{E}$ apoprotein due to cysteine-arginine interchange at a single site. J. Biol. Chem. 257:2518-2521.

11. Schneider, W. J., P. T. Kovanen, M. S. Brown, J. L. Goldstein, G. Utermann, W. Weber, R. J. Havel, L. Kotite, J. P. Kane, T. L. Innerarity, and R. W. Mahley. 1981. Familial dysbetalipoproteinemia: abnormal binding of mutant apoprotein $\mathrm{E}$ to low density lipoprotein receptors of human fibroblasts and membranes from liver and adrenal of rats, rabbits, and cows. J. Clin. Invest. 68:1075-1085.

12. Rall, S. C., Jr., K. H. Weisgraber, T. L. Innerarity, and R. W. Mahley. 1982. Structural basis for receptor binding heterogeneity of apolipoprotein E from type III hyperlipoproteinemic subjects. Proc. Natl. Acad. Sci. USA. 79:4696-4700.

13. Rall, S. C., Jr., K. H. Weisgraber, T. L. Innerarity, T. P. Bersot, R. W. Mahley, and C. B. Blum. 1983. Identification of a new structural variant of human apolipoprotein E, E2(Lys ${ }_{146} \rightarrow$ Gln), in a type III hyperlipoproteinemic subject with the $\mathrm{E} 3 / 2$ phenotype. J. Clin. Invest. 72:1288-1297.

14. Utermann, G., U. Langenbeck, U. Beisiegel, and W. Weber. 1980. Genetics of the apolipoprotein E system in man. Am. J. Hum. Genet. 32:339-347.

15. Zannis, V. I., and J. L. Breslow. 1981. Human very low density lipoprotein apolipoprotein $\mathrm{E}$ isoprotein polymorphism is explained by genetic variation and posttranslational modification. Biochemistry. 20:1033-1041.

16. Utermann, G., A. Steinmetz, and W. Weber. 1982. Genetic control of human apolipoprotein E polymorphism: comparison of oneand two-dimensional techniques of isoprotein analysis. Hum. Genet. 60:344-351.

17. Ghiselli, G., E. J. Schaefer, L. A. Zech, R. E. Gregg, and H. B. Brewer, Jr. 1982. Increased prevalence of apolipoprotein $E_{4}$ in type $V$ hyperlipoproteinemia. J. Clin. Invest. 70:474-477.

18. Yamamura, T., A. Yamamoto, K. Hiramori, and S. Nambu. 1984. A new isoform of apolipoprotein E-apo E-5-associated with hyperlipidemia and atherosclerosis. Atherosclerosis. 50:159-172.

19. Yamamura, T., H. Sudo, K. Ishikawa, and A. Yamamoto. 1979. Familial type I hyperlipoproteinemia caused by apolipoprotein C-II deficiency. Atherosclerosis. 34:53-65.

20. Burstein, M., and H. R. Scholnick. 1973. Lipoprotein-polyanionmetal interactions. Adv. Lipid Res. 11:67-108.

21. Lowry, O. H., N. J. Rosebrough, A. L. Farr, and R. J. Randall. 1951. Protein measurement with the Folin phenol reagent. J. Biol. Chem. 193:265-275.

22. Kashyap, M. L., B. A. Hynd, and K. Robinson. 1980. A rapid and simple method for measurement of total protein in very low density lipoproteins by the Lowry assay. J. Lipid Res. 21:491-495.

23. Warnick, G. R., C. Mayfield, J. J. Albers, and W. R. Hazzard. 
1979. Gel isoelectric focusing method for specific diagnosis of familial hyperlipoproteinemia type 3. Clin. Chem. 25:279-284.

24. Laemmli, U. K. 1970. Cleavage of structural proteins during the assembly of the head of bacteriophage T4. Nature (Lond.). 227:680685.

25. O'Farrell, P. H. 1975. High resolution two-dimensional electrophoresis of proteins. J. Biol. Chem. 250:4007-4021.

26. Shelburne, F. A., and S. H. Quarfordt. 1977. The interaction of heparin with an apoprotein of human very low density lipoprotein. J. Clin. Invest. 60:944-950.

27. Weber, K., and M. Osborn. 1969. The reliability of molecular weight determinations by dodecyl sulfate-polyacrylamide gel electrophoresis. J. Biol. Chem. 244:4406-4412.

28. Fainaru, M., R. W. Mahley, R. L. Hamilton, and T. L. Innerarity. 1982. Structural and metabolic heterogeneity of $\beta$-very low density lipoproteins from cholesterol-fed dogs and from humans with type III hyperlipoproteinemia. J. Lipid Res. 23:702-714.
29. Menzel, H.-J., R.-G. Kladetzky, and G. Assmann. 1983. Apolipoprotein E polymorphism and coronary artery disease. Arteriosclerosis. 3:310-315.

30. Innerarity, T. L., E. J. Friedlander, S. C. Rall, Jr., K. H. Weisgraber, and R. W. Mahley. 1983. The receptor-binding domain of human apolipoprotein E: binding of apolipoprotein $\mathrm{E}$ fragments. $J$. Biol. Chem. 258:12341-12347.

31. Weisgraber, K. H., T. L. Innerarity, K. J. Harder, R. W. Mahley, R. W. Milne, Y. L. Marcel, and J. T. Sparrow. 1983. The receptor-binding domain of human apolipoprotein E: monoclonal antibody inhibition of binding. 1983. J. Biol. Chem. 258:12348-12354.

32. Zannis, V. I., J. L. Breslow, G. Utermann, R. W. Mahley, K. H. Weisgraber, R. J. Havel, J. L. Goldstein, M. S. Brown, G. Schonfeld, W. R. Hazzard, and C. Blum. 1982. Proposed nomenclature of apoE isoproteins, apoE genotypes, and phenotypes. J. Lipid Res. 23:911-914. 